\title{
TUBERIAS de PVC ORIENTADO: EFICIENCIA, AHORRO ENERGÉTICO y SOSTENIBILIDAD
}

\author{
Martínez del Amo, Y (1) (P), Romero Montoya, M (2) \\ ${ }^{1}$ Director Calidad y Producto, Molecor Tecnología, S.L., yolanda.martinez@molecor.com \\ ${ }^{2}$ Técnico Infraestructuras Hidráulicas, Molecor Tecnología, S.L, manel.romero@molecor.com
}

\section{Resumen}

Ante la problemática del calentamiento global que sufre el planeta, muchas administraciones y gestoras de agua, están planeando el uso de materiales comprometidos con el medioambiente, buscando soluciones ecológicas y favorables con el desarrollo sostenible del planeta. Las tuberías molecularmente orientadas (PVC-O), se utilizan cada vez con más frecuencia en obras de canalizaciones de agua a presión, por ser la solución actual de mayor eficiencia en la gestión de recursos hidráulicos que demandan las infraestructuras modernas. La confianza en el uso mundial de este tipo de tuberías, se debe, a sus excepcionales propiedades físico-mecánicas perdurables en el tiempo, así como a su inalterabilidad química, ambos indicadores ampliamente ventajosos respecto a las canalizaciones tradicionales. A esto hay que unir, su compromiso con el medio ambiente, siendo la tubería más ecológica del mercado por la menor huella ambiental que presentan.

\section{1- Introducción}

Los factores que determinan la eficiencia energética durante todo el ciclo de vida de una tubería son principalmente, el tipo de materia prima utilizada, el proceso de producción, el acabado del producto y su vida útil.

Las tuberías de PVC Orientado, evitan el consumo innecesario de energía a lo largo de toda su larga vida útil, ya que analizados todos sus parámetros, ofrece mejores resultados respecto al resto de materiales, siendo esto confirmado por estudios y modelizaciones de prestigio internacional.

Según el informe de la Comisión Europea (Abril 2004) "Life Cycle Assessment (LCA) of PVC and of principal competing materials", el ciclo de vida para los estos productos, incluida la tubería orientada, es el mostrado en la figura 1.

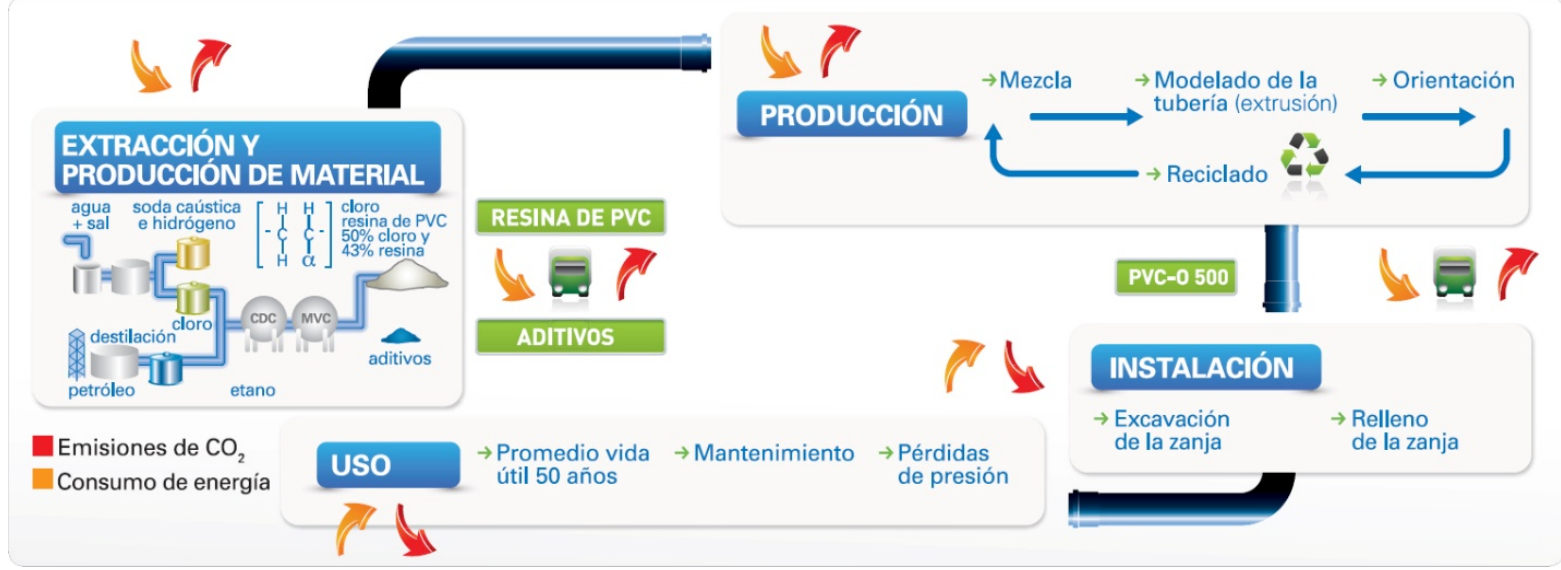

Figura 1. Ciclo de vida de las tuberías de PVC-O 
Siguiendo el esquema del proceso descriptivo, se muestran la eficiencia, el ahorro energético y la sostenibilidad en todas las etapas de su ciclo de vida.

- Fase producción.

- Fase de uso.

- Fase de reciclaje o disposición final.

\section{2- Eficiencia en materias primas y en el proceso productivo}

Las tuberías de PVC-O son fabricadas por extrusión convencional y posteriormente sometidas a un proceso de orientación molecular. El tubo base extruido, bajo unas determinadas condiciones de temperatura y presión, es sometido a un estiramiento que produce en el interior de la tubería la reordenación de las moléculas del polímero, formando una estructura laminar (figura 2), que mejora de forma significativa las propiedades mecánicas del producto, a la vez que mantiene intactas sus propiedades químicas.

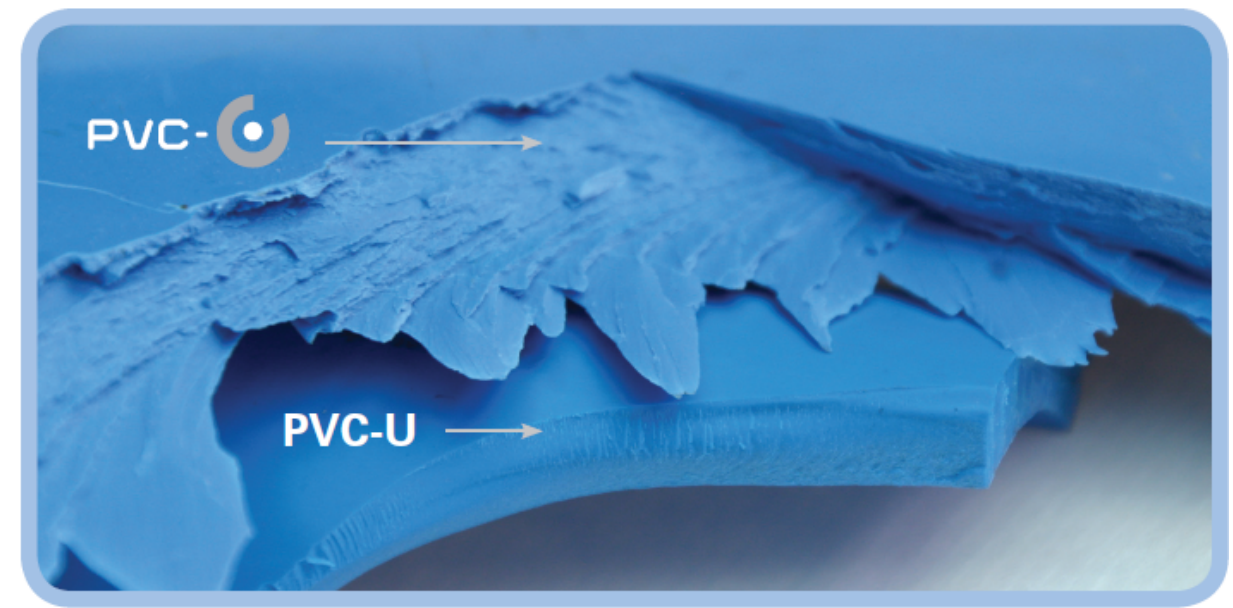

Figura 2 Estructura laminar del PVC-O vs estructura amorfa del PVC convencional

Al mismo tiempo que se obtiene esta mejora de propiedades, tiene lugar una optimización del espesor de la pared del tubo. Así racionalizando el uso de materias primas, a la vez que consiguen tubos de mejores prestaciones, se produce una mayor eficacia en el uso de los recursos.

Este menor consumo de materias primas, se traduce en ahorro de energía durante la extracción de las mismas y la producción del material.

\section{3- Eficiencia durante el uso}

La vida útil de una tubería para el transporte de agua debe garantizar un periodo de 50 años, según establecen las curvas de regresión que determinan su tensión máxima de diseño, pero puede confirmarse que instalaciones en uso de tuberías plásticas analizadas y ensayadas bajo el rigor de las exigencias normativas actuales, prevé un servicio en explotación superior a 100 años para esta modalidad de canalizaciones de nueva generación. Esta vida útil, comienza en el momento de la construcción de la red con la instalación de la tubería. En esta fase, el consumo de energía más importante se debe al bombeo. 
Al respecto de la eficiencia y el ahorro energético que tiene lugar durante esta etapa, se pueden diferenciar varios aspectos:

\section{- Optimización del transporte}

Gracias a su ligereza, podemos transportar más cantidad de material, por tanto tenemos ahorro de combustible y minimización de las emisiones de $\mathrm{CO}_{2}$.

\section{- Eficiencia en costes de instalación}

- Su ligereza también minimiza la repercusión de la mano obra y la necesidad de maquinaria especial para su puesta en obra.

- El eficaz diseño de la copa permite que los tubos sean ensamblados con gran facilidad.

Uniendo estos dos factores, se consigue un rendimiento de instalación muy alto, reduciéndose de forma considerable los tiempos de ejecución de las obras, obteniéndose beneficios económicos y sociales.

Teniendo en cuenta también, que el coste de la tubería ofrece ventajas competitivas, cuando es comparado con otros materiales, el coste total de la conducción puede verse reducido sensiblemente, según el material y los diámetros a instalar, con la consiguiente optimización de recursos económicos que pueden ser destinados a la construcción de nuevas instalaciones (figura 3).

\section{COSTE DE LA INSTALACIÓN}

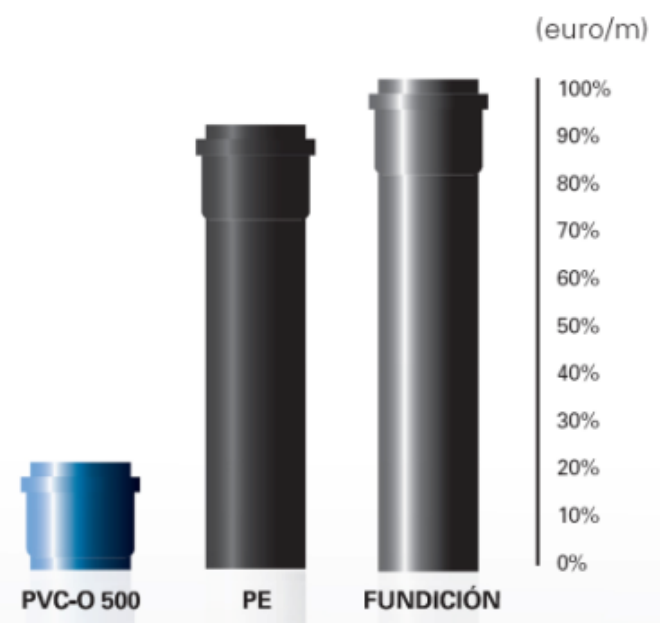

Figura 3 Gráfico costes de instalación de los distintos tipos de tuberías

\section{- Eficiencia durante el funcionamiento}

Este tipo de tuberías, presentan una mayor capacidad hidráulica, de hasta el $40 \%$, dependiendo del material y el diámetro con que se comparen, con lo que para un mismo consumo energético, siempre la cantidad de agua transportada es mayor.

La extremada lisura de esta superficie interior (figura 4), hace que la formación de depósitos sea difícil, por lo que el diámetro interior apenas se ve reducido a lo largo del tiempo y así el caudal del fluido para el que la tubería ha sido diseñada se mantendrá invariable durante su utilización. 


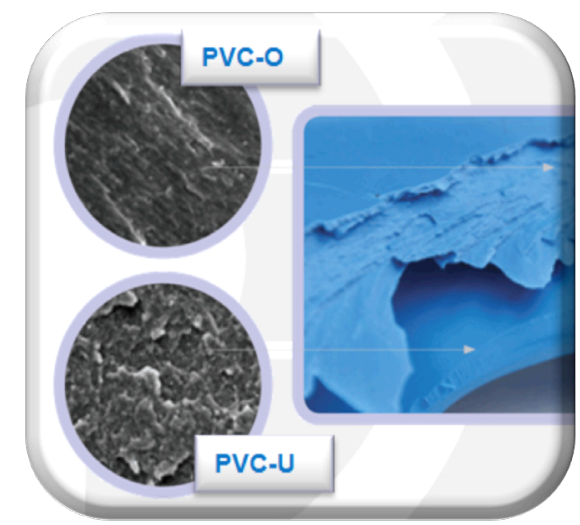

Figura 4 Rugosidad PVC-O vs PVC-U

Este aumento de capacidad hidráulica se debe a su mayor sección útil (figura 5), ventaja debida a las propiedades que presentan las canalizaciones molecularmente orientadas. Por otra parte, su menor rugosidad hace que la superficie interna del tubo sea extremadamente lisa, con lo que se minimizan las pérdidas de carga y el fluido puede circular a mayor velocidad.

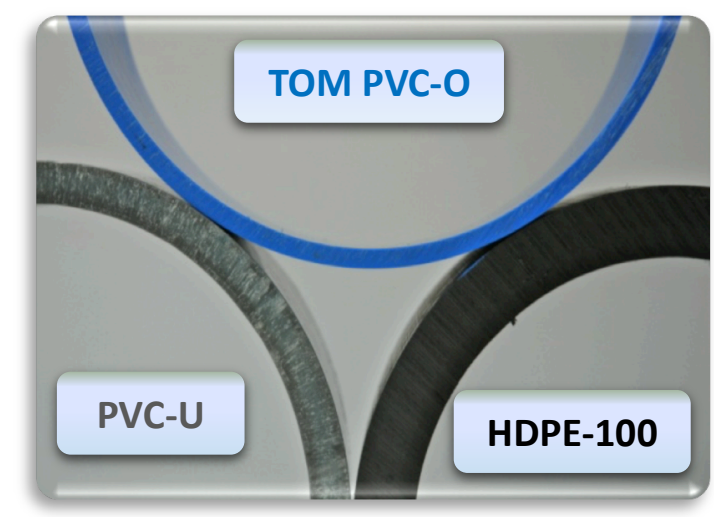

Figura 5 Comparativa de espesores

Todos estos beneficios hacen que para transportar la misma cantidad de agua, se necesite menos energía, de esta forma, se reducen los consumos energéticos en el bombeo durante la etapa de uso (figura 6).

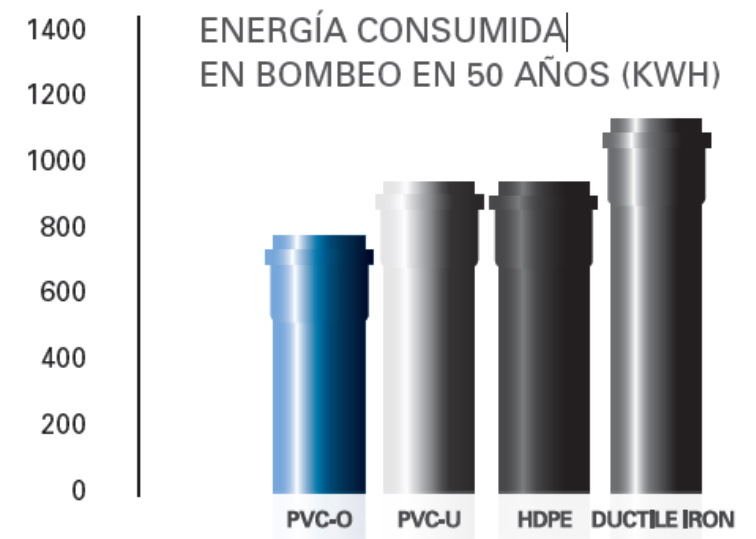

Figura 6 Gráfico energía consumida durante la fase de bombeo de distintos materiales 
En el caso de las tuberías de PVC-O, según el estudio "Estimación del consumo energético y de la emisión de $\mathrm{CO}_{2}$ asociada a la producción, uso y disposición final de tuberías de PVC, PEHD, PP, Fundición y Hormigón" realizado por la Universidad Politécnica de Catalunya (figura 7), se obtiene el mejor comportamiento tanto en el menor consumo energético, como en la menor emisión de $\mathrm{CO}_{2}$ a la atmósfera, por lo que su contribución al efecto invernadero es menor. Todo esto hace que, las tuberías de PVC-O sean la solución más ecológica debido a su mejor contribución al correcto desarrollo sostenible del planeta.

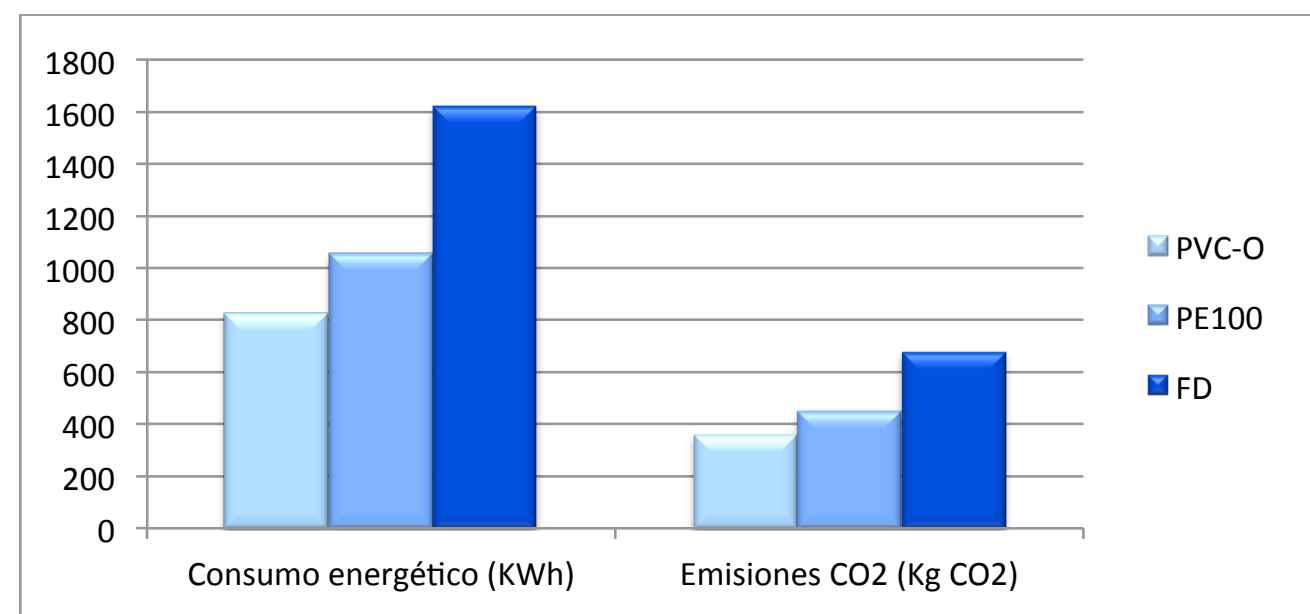

Figura 7 Gráfico energía consumida y emisiones de $\mathrm{CO}_{2}$ durante todo el ciclo de vida de un sistema de tubería

\section{- Elevada vida útil}

La estructura laminar obtenida durante el proceso de orientación molecular que tiene lugar en el proceso de su fabricación, confiere a las tuberías de PVC-O una mejora significativa de sus propiedades mecánicas (figura 8), resultando una canalización con una gran resistencia al impacto y a cargas externas, mejorando su resistencia a tracción y eliminando el riesgo de una propagación de fisura rápida debido a su morfología ordenada.

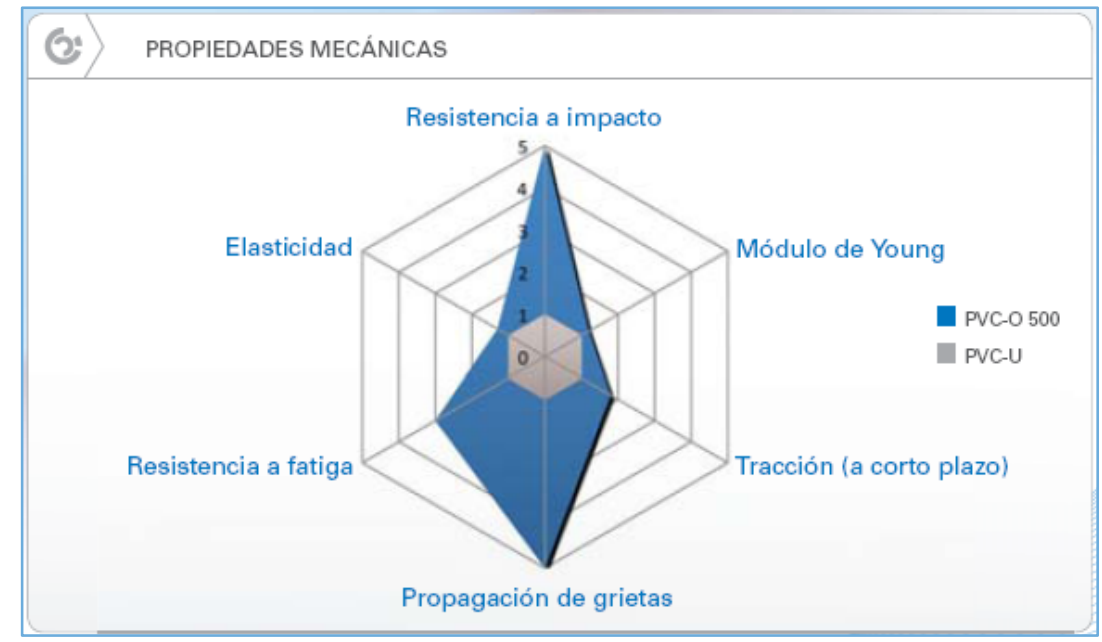

Figura 8 Comparativa propiedades mecánicas del PVC-o vs al PVC-U

Gracias a estas excelentes propiedades mecánicas, la tubería es altamente resistente y permanece inalterada durante años, con lo que se minimizan de forma 
significativa, las sustituciones de tuberías dañadas o deterioradas en la red con el consiguiente ahorro de recursos humanos y económicos.

\section{5- Eficiencia en la gestión de residuos}

EI PVC es un material $100 \%$ reciclable, puede ser reutilizado tras una larga vida de servicio y mediante un proceso de bajo coste, mayoritariamente mecánico. El proceso de fabricación de las tuberías molecularmente orientadas, por su alto requerimiento técnico, no permite la utilización de materiales reciclados, pero permiten un proceso de gestión integral, ya que puede ser utilizadas, tras su ciclo de vida, en la fabricación de otras aplicaciones plásticas, como el recubrimiento de cables o la producción de bajantes para evacuación, donde los requerimientos técnicos exigidos a la materia prima son menores.

De esta forma, se reduce el consumo de materias primas vírgenes y también el volumen de los residuos generados.

El fomento de una cultura de reutilización y reciclaje, queda reflejado en el informe de la Comisión Europea de Abril 2004 y del compromiso voluntario de los actores del sector en la gestión de los residuos a nivel Europeo en sus programas Vinyl2010 y VinylPlus.

\section{6- Optimización de recursos hídricos}

Las redes de suministro de agua fabricadas con materiales tradicionales, registran actualmente, un elevado porcentaje de fugas del agua canalizada, debido tanto a la falta de estanqueidad de las uniones, como a las roturas producidas por el deterioro de los tubos. En muchos casos, esto lleva a que las canalizaciones tengan que ser reemplazadas pocos años después de ser instaladas.

Las tuberías orientadas, se presentan como una garantía de durabilidad y compromiso para la conservación de los recursos hídricos aportando soluciones a infraestructuras en equilibrio con los ecosistemas. Los parámetros que contribuyen a su valoración ecopositiva son:

- La completa fiabilidad de las uniones, gracias a una junta elástica de alta calidad y a un eficaz diseño de la copa, que asegura la completa estanqueidad de la conducción, evitando fugas del agua canalizada.

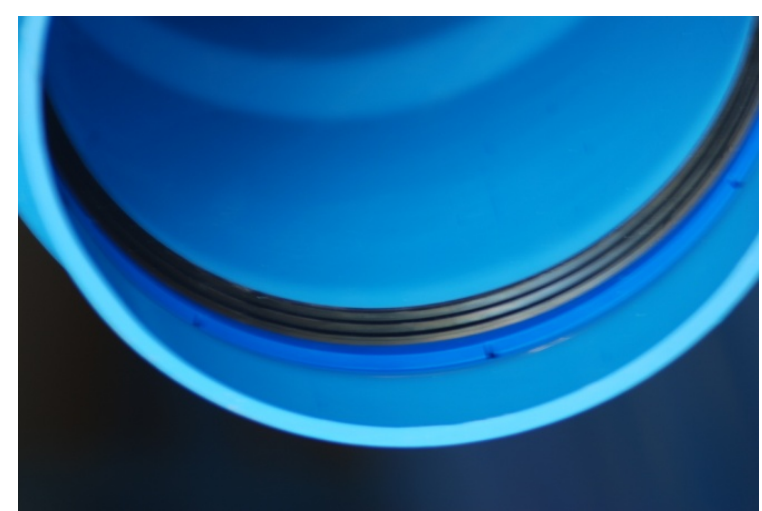

Figura 9 Detalle de la junta de estanqueidad de una tubería TOM@ de PVC-O

- La inercia química del PVC que hace que las tuberías permanezcan inalteradas durante toda su vida útil. De esta forma:

- Es inerte a fenómenos cinéticos de corrosión electroquímica, bacteriana, fisurante y por fatiga, que producen los componentes del suelo natural. Por lo que: 
- No hay que preocuparse por la naturaleza del suelo donde vayan a ser enterradas las tuberías.

- No se necesitan recubrimientos de protección, ni exteriores ni interiores.

- La tubería al no ser afectada por la corrosión, evita la cesión de óxidos y metales al agua, que en caso de agua potable, pueden llegar a suponer un problema de salud pública si se superan los parámetros de calidad exigidos para el agua de consumo humano.

- Es muy resistente frente a una amplia variedad de productos químicos, como desinfectantes o productos fitosanitarios, utilizados en los distintos sectores.

- Es resistente a los biocidas, oxidantes presentes de forma habitual en aguas potables y en los procesos de erradicación de flora y faunas invasoras, sin alterar sus propiedades.

- Su alta resistencia química hace que no se produzca deterioro de la pared del tubo, que en otros materiales puede ocasionar agujeros y fisuras que permitan la introducción de agentes externos en el caudal del agua transportada provocando su contaminación.

- No favorece el crecimiento de microorganismos.

\section{9- Conclusiones}

Gracias a la orientación molecular, las tuberías de PVC-O presentan ventajas aplicables tanto a la calidad del producto y a sus propiedades mecánicas, como al mayor rendimiento de instalación, así se consigue una eficiencia en costes en la ejecución de las redes de riego.

No menos importante es su mejor contribución, durante todo su largo ciclo de vida, al correcto desarrollo sostenible del planeta y a la optimización del consumo de recursos naturales.

\section{- Eficiencia}

- Menor consumo de materias primas: Petróleo y PVC

- Menos residuos y además $100 \%$ reciclable

- Larga vida útil

\section{- Ahorro}

- Menor consumo de energía a lo largo de todo su ciclo de vida: fabricación, transporte, instalación, uso (bombeo) y disposición final

- Optimización de recursos hídricos

\section{- Sostenibilidad}

- Mejor huella ambiental que otros materiales tradicionales

- Menor contribución al calentamiento global 